\title{
CONTRIBUTION A LA CONNAISSANCE DE LA FLORE FONGIQUE KÉRATINOPHILE ISOLÉE DES PETITS MAMMIFÈRES SAUVAGES ET DU LAPIN DE GARENNE EN FRANCE
}

\author{
Discussion sur les espèces fongiques rencontrées
}

\author{
D. CHABASSE*, C. GUIGUEN**, A. COUATARMANAC'H**, \\ H. LAUNAY***, V. REECHT*, C. DE BIEVRE****
}

RÉSUME. La présence de dermatophytes ou de champignons kératinophiles assimilés fut recherchée chez 237 petits mammifères sauvages et 125 lapins de garenne. Le but principal de ce travail est de vérifier quelles espèces de champignons existent sur ces animaux. Quatre espèces de dermatophytes furent isolées : Trichophyton ajelloi, Trichophyton terrestre, Trichophyton mentagrophyles, Microsporum persicolor. Trichophyton terrestre est le plus fréquemment isolé, si fréquemment que sa présence ne peut être expliquée par une simple contamination à partir du sol. Le genre Chrysosporium est bien représenté chez beaucoup de ces animaux : Chrysosporium keratinophilum, Chrysosporium tropicum, Chrysosporium muttifidum, Chrysosporium pannorum, Chrysosporium georgii, Chrysosporium merdarium, Chrysosporium anamorphe d'Arthroderma curreyi, Chrysosporium anamorphe d'Arthroderma cuniculi, Anixiopsis stercoraria, Chrysosporium parvum.

Les petits mammifères sauvages et le lapin de garenne en France se comportent, non seulement comme des vecteurs de ces espèces kératinophiles, dermatophytes et assimilés, mais aussi apportent un environnement favorable au maintien de leur "état "saprophytique. La mise en évidence de nombreuses espèces issues de leur pelage isolées pour la première fois en France constitue une observation qui mérite d'être soulignée.

Mots-clés : Champignon kératinophile. Dermatophytes. Chrysosporium. Petits mammifères sauvages. Lapin de garenne. France.

\section{Contribution to the knowledge of keratinophilic fungi isolated from small wild mammals and european rabbit in France, discussion about fungal species found.}

SUMMARY. The occurrence of dermatophytes and other keratinophilic fungi was investigated in 237 small wild mammals and 125 european rabbit. The purpose of the investigation was to determine what were the species of fungi present in the these animals. Four species of dermato-

* Service des Maladies Parasitaires et Exotiques (Pr P. Hocquet), C. H. U., 4, rue Larrey, F 49033 Angers Cedex.

** Laboratoire de Parasitologie, Faculté de Médecine et de Pharmacie, avenue du Professeur Léon-Bernard, F 35043 Rennes Cedex. Cedex.

*** Institut de Parasitologie de l'Ouest, avenue du Professeur Léon-Bernard, F 35043 Rennes

*** Unité de Mycologie, Institut Pasteur, 28, rue du Dr Roux, F 75724 Paris Cedex 15.

Accepté le 6 décembre 1986. 
phytes were isolated: Trichophyton ajelloi, Trichophyton terrestre, Trichophyton mentagrophytes, Microsporum persicotor. Trichophyton terrestre was the most frequently isolated and it occurred more frequently than its presence could be explained by the contamination from soil. Members of the genus Chrysosporium were found in many animals: Chrysosporium keratinophilum, Chrysosporium tropicum, Chrysosporium multifudum, Chrysosporium pannorum, Chrysosporium georgii, Chrysosporium merdarium, Chrysosporium anamorph of Arthroderma curreyi, Chrysosporium anamorph of Arthroderma cuniculi, Anixiopsis stercoraria, Chrysosporium parvum.

Wild small mammals and european rabbits in France, not only act as carrier of keratinophilic fungi and allied dermatophytes but also provide a suitable habitat for their survival as saprophytes. The recurrence of numerous species present on the coat, isolated fort the first time in France was remarkable.

Key-words : Keratinophilic fungi. Dermatophytes. Chrysosporium. Small wild mammals. European rabbit (L). France.

\section{Introduction}

Les petits mammifères sauvages sont connus comme étant une source possible d'infestation par les champignons kératinophiles, en particulier des dermatophytes, pour l'Homme comme le témoignent nombreux travaux (Menges et coll., 1957 ; McKeever et coll., 1958 ; English et Bayley, 1978 ; English et coll., 1967 ; Alteras et coll., 1966 ; Marples, 1967 ; Chmel et Buchwald, 1967 ; Otcenasek et Dvorak, 1952 ; Otcenasek et coll., 1980 ; Knudtson et Roberstad, 1970 ; Gugnani et coll., 1978 ; Battelli et coll., 1978 ; Hubalek et coll., 1979 ; Mantovani et coll., 1982).

En France, de semblables recherches ont été entreprises (Badillet et coll., 1972 ; Houin et coll. en Franche-Comté, 1972 ; Mariat et coll. en Alsace, 1976). A l'inverse, le rôle épidémiologique du lapin de garenne (Oryctolagus cuniculus) dans la transmission des kératinophiles pathogènes a été peu étudié en France (Albala et Moreda, 1981 ; Beaucournu et Launay, 1977).

Dans le cadre d'enquêtes entomologiques et virologiques menées depuis plusieurs années dans l'Ouest de la France, principalement avec les laboratoires de Parasitologie de la Faculté de Médecine de Rennes et celui de Virologie de la Faculté de Médecine de Brest, nous rapportons et commentons dans ce travail qui a fait l'objet de deux brèves Communications préliminaires (Chabasse et coll., 1984, 1986) ; les résultats mycologiques concernant la flore kératinophile isolée du pelage de 362 animaux capturés.

\section{Matériel et méthodes}

\section{LE PIÉGEAGE}

\subsection{Petits mammifères sauvages.}

Apodemus sylvaticus Linne 1758 (mulot sylvestre), Clethrionomys glareolus Schreber 1780 (campagnol roussâtre), Crocidura russula Hermann 1780 (musa- 
raigne musette) Sorex araneus Linne 1758 (musaraigne carrelet) ; Talpa europaea Linne 1758 (taupe) ont été piégés.

a) Dans la réserve "Michel-Hervé-Julien " du cap Sizun (Finistère) les 3 et 4 novembre 1983 et les 5 et 6 mai 1984. La végétation y est faite de broussailles et d'herbes hautes, le relief au sol constitué de micaschistes est accidenté, le climat $\mathrm{y}$ est très humide et l'influence de la mer y est importante.

b) Sur le plateau calcaire de Saulges à l'est de Laval en février 1982 et en mai 1984, la couverture végétale est riche, constituée de ronces, genévriers, sorbiers, ajoncs avec une grande abondance de buis.

c) Dans le Cantal près d'Allanche et Monboudif à $1000 \mathrm{~m}$ d'altitude environ, les 21, 22, 23 mai 1985.

A chaque fois, 100 pièges de type Shermann et une vingtaine de pièges à taupes sont posés pendant deux nuits consécutives. Les Shermann sont placés dans les zones couvertes (talus, ronciers, ajoncs...) après recherche d'indices de fréquentation quand cela est possible (terriers, crottes...) et utilisation d'un appât (croûton) mis au fond de chaque piège. Le lendemain les pièges sont relevés et l'animal piégé est immédiatement transféré dans un sac en plastique où il est sacrifié. Les pièges à taupe placés la veille dans les galeries tuent celles-ci dès leur capture.

\subsection{Les lapins de garenne.}

Ils proviennent, soit du parc de l'Arboretum du Muséum National d'Histoire Natuı elle de Paris à Chèvreloup dans les Yvelines, soit du parc breton de Ploumagoar près de Guingamp (Côtes-du-Nord). Ils ont été capturés par battues aux chiens courants, avec prise au filet et par furetage. Les prélèvements ont eu lieu en mai, septembre, octobre 1984 et en février et mars 1985. 125 animaux ont été ainsi étudiés.

\section{LES PRÉLÈVEMENTS}

2.1. A partir de carrés de moquette de $2 \mathrm{~cm}^{2}$ stérilisés à l'autoclave à $120^{\circ} \mathrm{C}$ pendant une heure, pour chaque animal une moquette est passée à rebrousse poil sur le dos, les flancs et la face ventrale puis remise dans un emballage stérile.

2.2. Avec une pince à épiler stérilisée à chaque fois à la flamme, on retire des poils principalement derrière les oreilles, qui sont ensuite déposés dans une boîte de Pétri stérile pour le transfert. Les échantillons sont conservés quelques jours à $+44^{\circ} \mathrm{C}$.

\section{L'ENSEMENCEMENT}

Il est réalisé au laboratoire moins de 10 jours après le prélèvement sur le "terrain " utilisant le milieu de base de Sabouraud additionné de $1 \mathrm{~g}$ de Chloramphénicol et de $1 \mathrm{~g}$ de Cycloheximide par litre.

Les carrés de moquettes sont appliqués et légèrement secoués à la surface 
de la gélose, tandis que les poils sont ensemencés séparément. Les boîtes de Pétri sont ensuite déposées à l'étuve à $25^{\circ} \mathrm{C}$ et examinées tous les 3 jours.

\section{Discussion}

1. Valeur de l'association des 2 techniques de prélèvements pour l'isoleMENT DES KÉRATINOPHILES

Sur 237 petits mammifères sauvages examinés, 192 d'entre eux sont porteurs d'au moins une espèce kératinophile, soit $81 \%$. Cette fréquence est plus élevée encore avec les lapins de garenne, 93,6\% (tableau $I$ ). En associant le prélèvement. de poils à la technique de référence (le prélèvement sur moquette) (Mariat et coll., 1976), le nombre de souches fongiques isolées est plus élevé.

Tableau 1. - Valeur de la technique de prélèvements dans l'isolement des kératinophiles.

\begin{tabular}{|c|c|c|c|c|c|c|}
\hline \multirow{2}{*}{$\begin{array}{c}\text { Petits } \\
\text { mammifères } \\
\text { sauvages }\end{array}$} & \multirow{2}{*}{ 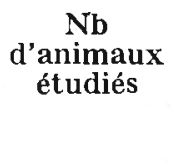 } & \multirow{2}{*}{$\underset{\substack{\text { d'animaux } \\
\text { positifs }}}{\text { ding }}$} & \multicolumn{2}{|c|}{$\begin{array}{l}\text { Nb de souches } \\
\text { isolées sur }\end{array}$} & \multirow{2}{*}{$\begin{array}{c}\mathrm{Nb} \\
\text { de souches } \\
\text { différentes } \\
\text { isolées } \\
\text { sur } \mathbf{M}+\mathrm{P}\end{array}$} & \multirow{2}{*}{$\begin{array}{c}\text { Gain } \\
\mathbf{M}+\mathbf{P} \\
\text { par } \\
\text { rapport } \\
\text { a } M\end{array}$} \\
\hline & & & $\begin{array}{l}\text { moquette } \\
\text { (M) }\end{array}$ & $\begin{array}{c}\text { poils } \\
(\mathrm{P})\end{array}$ & & \\
\hline $\begin{array}{l}\text { Apodemus } \\
\text { sylvaticus }\end{array}$ & 146 & 116 & 124 & 115 & 181 & +57 \\
\hline $\begin{array}{l}\text { Clethriono- } \\
\text { mys } \\
\text { glareolus }\end{array}$ & 51 & 46 & 60 & 54 & 84 & +24 \\
\hline $\begin{array}{l}\text { Crocidura } \\
\text { russula }\end{array}$ & 25 & 20 & 19 & 16 & 30 & +11 \\
\hline $\begin{array}{l}\text { Sorex } \\
\text { coronatus }\end{array}$ & 2 & 2 & 3 & 4 & 4 & +1 \\
\hline $\begin{array}{c}\text { Sorex } \\
\text { araneus }\end{array}$ & 1 & 1 & 1 & 0 & 1 & +0 \\
\hline $\begin{array}{l}\text { Talpa euro- } \\
\text { paea }\end{array}$ & 12 & 8 & 6 & 7 & 12 & +6 \\
\hline $\begin{array}{l}\text { Total } \\
\text { Oryctolagus } \\
\text { cuniculus }\end{array}$ & $\begin{array}{l}237 \\
125\end{array}$ & $\begin{array}{l}192(81 \%) \\
117(93,6 \%)\end{array}$ & $\begin{array}{l}213 \\
204\end{array}$ & $\begin{array}{l}196 \\
131\end{array}$ & $\begin{array}{l}312 \\
301\end{array}$ & $\begin{array}{r}+99 \\
+97\end{array}$ \\
\hline Total & 362 & $309(85,3 \%)$ & 417 & 327 & 613 & +196 \\
\hline
\end{tabular}

2. FréQuence et répartition des kératinophiles (DERMatophytes et espèces ASSIMILÉES) ISOLÉS CHEZ LES ANIMAUX ÉTUdIÉS

La flore kératinophile est importante et variée, 15 espèces différentes ont pu être identifiées dont 4 dermatophytes. Le genre Chrysosporium, proche des dermatophytes avec 10 espèces difféı entes est bien représenté (tableau II). 
Tableau II. - Fréquence et répartition

des champignons kératinophiles chez les animaux étudiés.

\begin{tabular}{|c|c|c|c|c|c|c|}
\hline & $\begin{array}{l}\text { Oryctolagus } \\
\text { cunicutus }\end{array}$ & $\begin{array}{l}\text { Apodemus } \\
\text { sylvaticus }\end{array}$ & $\begin{array}{l}\text { Clethriono- } \\
\text { mys } \\
\text { glareolus }\end{array}$ & $\begin{array}{l}\text { Croci- } \\
\text { dura } \\
\text { russula }\end{array}$ & $\begin{array}{l}\text { Sorex } \\
\mathrm{sp}\end{array}$ & $\begin{array}{l}\text { Talpa } \\
\text { euro- } \\
\text { paea }\end{array}$ \\
\hline $\begin{array}{l}\text { Nombre d'ani- } \\
\text { maux étu- } \\
\text { diés }\end{array}$ & 125 & 146 & 51 & 25 & 3 & 12 \\
\hline $\begin{array}{l}T . \text { ajelloï } \\
T . \text { terrestre } \\
T . \text { mentagro- } \\
\quad \text { phytes }\end{array}$ & $\begin{array}{c}2(1,6 \%) \\
93(74,4 \%) \\
0\end{array}$ & $\begin{array}{l}0 \\
61(41,8 \%) \\
4(2,7 \%)\end{array}$ & $\underset{\mathbf{0}}{\mathbf{0}}(39,2 \%)$ & $\begin{array}{l}0 \\
4(16 \%) \\
0\end{array}$ & $\begin{array}{l}0 \\
2 \\
0\end{array}$ & $\begin{array}{l}4 \\
3 \\
2\end{array}$ \\
\hline $\begin{array}{l}\text { M. persicolor } \\
C . \quad \text { keratino- } \\
\text { philum }\end{array}$ & $\begin{array}{l}0 \\
30(24 \%)\end{array}$ & $\begin{array}{l}3(2 \%) \\
5(3,4 \%)\end{array}$ & $\begin{array}{l}7(13,7 \%) \\
6(11,8 \%)\end{array}$ & $\begin{array}{l}0 \\
2(8 \%)\end{array}$ & $\begin{array}{l}0 \\
1\end{array}$ & $\begin{array}{l}0 \\
2\end{array}$ \\
\hline 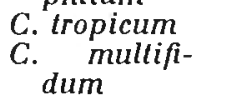 & $\begin{array}{l}2(1,6 \%) \\
6(4,8 \%)\end{array}$ & $\begin{array}{l}0 \\
0\end{array}$ & $\begin{array}{l}0 \\
0\end{array}$ & $\begin{array}{l}0 \\
0\end{array}$ & $\begin{array}{l}0 \\
0\end{array}$ & $\begin{array}{l}0 \\
0\end{array}$ \\
\hline $\begin{array}{l}C . \quad \text { anamor- } \\
\text { phe d'A. } \\
\text { curreyi }\end{array}$ & $37(29,6 \%)$ & $13(8,9 \%)$ & $11(26,6 \%)$ & $5(10 \%)$ & 0 & 1 \\
\hline $\begin{array}{l}\text { C. anamor- } \\
\text { phe d'A. } \\
\text { cuniculus }\end{array}$ & 0 & $8(5,5 \%)$ & $8(15,7 \%)$ & $3(12 \%)$ & 0 & 0 \\
\hline C. georgii & $40(32 \%)$ & $12(8,2 \%)$ & $2(3,9 \%)$ & 0 & 0 & 0 \\
\hline $\begin{array}{l}\text { C. pannorum } \\
\text { C. merda- }\end{array}$ & $\underset{0}{49}(39,6 \%)$ & $\begin{array}{c}37(25,3 \%) \\
2(1,3 \%)\end{array}$ & $\begin{array}{c}23(45 \%) \\
1(1,9 \%)\end{array}$ & $\begin{array}{l}9(36 \%) \\
0\end{array}$ & $\begin{array}{l}0 \\
0\end{array}$ & $\begin{array}{l}\mathbf{0} \\
0\end{array}$ \\
\hline $\begin{array}{l}\text { C. parvum } \\
\text { A. slercora- } \\
\text { ria }\end{array}$ & $\stackrel{0}{14}(8,8 \%)$ & $\begin{array}{l}2(1,3 \%) \\
3(2 \%)\end{array}$ & $\begin{array}{l}2(3,9 \%) \\
1(1,9 \%)\end{array}$ & $\begin{array}{l}1(4 \%) \\
0\end{array}$ & 0 & 0 \\
\hline $\begin{array}{l}\text { Chrysospo- } \\
\text { rium sp }\end{array}$ & $24(19,1 \%)$ & $22(15 \%)$ & $3(5,8 \%)$ & $2(8 \%)$ & 0 & 0 \\
\hline S. brevicaulis & $7(5,6 \%)$ & $9(6,1 \%)$ & 0 & 0 & $\mathbf{0}$ & 0 \\
\hline
\end{tabular}

\subsection{Les dermalophyles.}

2.1.1. Trichophyton terrestre, Durie et Frey, 1957.

Cette espèce décrite pour la première fois par Durie et Frey (1957), en Australie, est un dermatophyte isolé avec une grande fréquence chez les micromammifères sauvages (environ $40 \%$ des animaux piégés). Ceci confirme les travaux de Otcenasek et Dvorak (1962) et ceux de Dawson (1964) qui avait isolé T. terrestre sur $43,2 \%$ des micromammifères piégés alors que ce champignon n'était retrouvé que dans $12 \%$ des échantillons de sol. On peut noter à ce propos, que la présence de $T$. terrestre sur le pelage de ces mammifères, en particulier Clethrionomys glareolus, ne peut être expliquée par une simple contamination du sol à l'inverse de T. ajelloï qui, fréquemment isolé dans le sol, n'est que rarement rencontré sur ces animaux piégés (Dawson, 1964); Gentles et coll., 1965). 
TABleau III. - Fréquence de trichophyton terrestre chez les petits mammifères sauvages dans trois régions géographiques différentes.

\begin{tabular}{lccccc}
\hline & $\begin{array}{c}\text { 'Total } \\
\text { étu- } \\
\text { dié }\end{array}$ & $\begin{array}{c}\text { Animaux } \\
\text { positifs } \\
\text { avec } \\
\text { T. terrestre }\end{array}$ & Cap Sizun & Saulges & Cantal \\
\hline $\begin{array}{l}\text { Apodemus syl- } \\
\text { vaticus }\end{array}$ & 146 & $61(41,7 \%)$ & $47 / 81(58 \%)$ & $8 / 49(16,3 \%)$ & $6 / 16(37,5 \%)$ \\
$\begin{array}{l}\text { Clethrionomys } \\
\text { glareolus }\end{array}$ & 51 & $20(39,2 \%)$ & $6 / 12(50 \%)$ & $2 / 20(10 \%)$ & $12 / 9(63,2 \%)$ \\
\hline
\end{tabular}

Curieusement en France, dans l'étude de Mariat et coll. (1976), T. lerrestre n'est signalé que dans $0,21 \%$ des 3782 petits mammifères sauvages capturés et Houin et coll. (1972) isolent cette espèce chez 6 des 99 rongeurs examinés. La mise en culture tardive des prélèvements (moquette) était incriminée pour expliquer ce taux faible; nous ne confirmons pas cette hypothèse. Il ne semble pas non plus y avoir une spécificité bien nette de cette espèce avec un micromammifère bien déterminé. Si on compare les 3 zones géographiques étudiées dans notre enquête, les différences enregistrées sur la fréquence de T. terrestre (tableau III) ne dépendent pas de l'hòte Apodemus sylvaticus et Clethrionomys glareolus mais du lieu de piégeage. En effet, ce dermatophyte est moins fréquemment retrouvé sur le plateau calcaire de Saulges que dans la réserve bretonne du Cap Sizun ou des montagnes du Cantal $(\mathrm{P}<0,001)$. Ce sont chez les lapins de garenne que T. terrestre est isolé avec une plus grande fréquence, près de $75 \%$ des lapins prélevés, qu'ils soient issus de Chévreloup ( $80 \%$ ) ou capturés sur le Parc de Ploumagoar en Bretagne $(67 \%)$ (tableau IV). L'étude des courbes de répartition saisonnière de contamination de ce dermatophyte chez Oryclolagus cuniculus est plus délicate en raison de l'absence de capture en été mais si on compare les prélèvements en mai et octobre faits chez des lapins issus de Chèvreloup, la différence des isolats est significative $(\mathrm{P}<0,001)$ avec une contamination plus élevée en octobre; ceci mérite d'être

Tableau IV. - Fréquence de T. terrestre chez Oryctolagus cuniculus en fonction des dates de prélèvements.

\begin{tabular}{|c|c|c|c|c|c|c|c|}
\hline $\begin{array}{l}\text { Lieu } \\
\text { de } \\
\text { capture }\end{array}$ & $\begin{array}{c}\text { Total } \\
\text { des } \\
\text { animaux } \\
\text { examinés }\end{array}$ & $\begin{array}{c}\text { Total } \\
\text { des } \\
\text { positifs }\end{array}$ & $\begin{array}{l}5 \text { mai } \\
1984\end{array}$ & $\begin{array}{c}13 \text { sep- } \\
\text { tembre } \\
1984\end{array}$ & $\begin{array}{c}3 \text { octo- } \\
\text { bre } \\
1984\end{array}$ & $\begin{array}{l}2 \text { fé- } \\
\text { vrier } \\
1985\end{array}$ & $14 \underset{1985}{\text { mars }}$ \\
\hline $\begin{array}{l}\text { Chèvre- } \\
\text { loup }\end{array}$ & 60 & $\begin{array}{c}48 \\
(80 \%)\end{array}$ & $\begin{array}{c}5 / 15 \\
(33 \%)\end{array}$ & - & $\begin{array}{c}43 / 45 \\
(96 \%)\end{array}$ & - & - \\
\hline $\begin{array}{l}\text { Plouma- } \\
\text { goar }\end{array}$ & 65 & $\begin{array}{c}44 \% \\
(67 \%)\end{array}$ & - & $\begin{array}{l}11 / 20 \\
(55 \%)\end{array}$ & - & $\begin{array}{l}21 / 25 \\
(84 \%)\end{array}$ & $\begin{array}{l}12 / 20 \\
\left(60^{\circ}\right)\end{array}$ \\
\hline
\end{tabular}


confirmé. T. terrestre est dénué de pouvoir pathogène bien que Évolceanu et coll. (1962) réussissent à provoquer une mycose expérimentale chez la souris et que Scott et coll. (1980) lui attribuent une lésion chez un chien et un chat.

2.1.2. Microsporum persicolor, Sabouraud, 1910 et Trichophyton mentagrophytes, Sabouraud, 1893.

Ces dermatophytes ont été retrouvés essentiellement chez Clelhrionomys glareolus et Apodemus sylvaticus. Comme l'avait remarqué Badillet et coll. (1972) ; Houin et coll. (1972); English et Baylet (1972); Mariat et coll. (1976) (lableau V), les campagnols roussâtres sont contaminés préférentiellement par $M$. persicolor, mais nous ne retrouvons pas les chiffres élevés de contamination des rongeurs capturés dans certaines stations des îles britanniques (près de $60 \%$ ) (English et Southern, 1967). A l'inverse, T. menlagrophyles se retrouve plus volontiens sur Apodemus sylvaticus mais nous l'avons peu isolé. Les souches retrouvées chez Apodemus syllaticus et Talpa europaea correspondent à la variété astéroïdes présentant une couleur rouge groseille de l'envers des cultures. Si ces résultats confirment que le campagnol roussâtre est un réservoir non négligeable de $M$. persicolor, il faut noter malgré tout le nombre réduit de ces dermatophytes potentiellement pathogènes.

2.2. Les espèces voisines des dermatophyles.

Elles sont représentées essentiellement par le genre Chrysosporium, Carmichael, 1962. Ces champignons souvent kératinophiles et kératinolytiques,

TABlenu V. - Contamination de Clethrionomys glareolus et de Apodemus sylvaticus avec Microsporum persicolor et Trichophyton mentagrophytes.

\begin{tabular}{|c|c|c|c|c|c|}
\hline \multirow[t]{2}{*}{ Auteurs } & \multirow[t]{2}{*}{ Localités } & \multirow[t]{2}{*}{ Animaux } & \multirow{2}{*}{$\begin{array}{c}\text { Total } \\
\text { examiné }\end{array}$} & \multicolumn{2}{|c|}{ Infestation \% } \\
\hline & & & & $\begin{array}{l}\text { M. } \\
\text { persi- } \\
\text { color }\end{array}$ & $\begin{array}{c}T . \\
\text { menta- } \\
\text { grophytes }\end{array}$ \\
\hline $\begin{array}{l}\text { Badillet et coll., } \\
1972\end{array}$ & Grenoble, Isère & $\left\{\begin{array}{l}\text { C. glareolus } \\
\text { A. sylvaticus }\end{array}\right.$ & $\begin{array}{l}16 \\
15\end{array}$ & $\stackrel{25}{1,7}$ & $\begin{array}{l}0 \\
0\end{array}$ \\
\hline$\underset{1972}{\text { Houin et coll., }}$ & $\begin{array}{l}\text { Haute-Savoie, } \\
\text { Est de la } \\
\text { France }\end{array}$ & $\left\{\begin{array}{l}\text { C. glareolus } \\
\text { A. sylvaticus }\end{array}\right.$ & $\begin{array}{l}42 \\
40\end{array}$ & $\begin{array}{l}31 \\
15\end{array}$ & $\begin{array}{r}2 \\
43\end{array}$ \\
\hline $\begin{array}{l}\text { Mariat et coll., } \\
1976\end{array}$ & Alsace & $\left\{\begin{array}{l}\text { C. glareolus } \\
\text { A. sylvaticus }\end{array}\right.$ & $\begin{array}{l}1789 \\
1330\end{array}$ & $\begin{array}{c}11,63 \\
3,5\end{array}$ & $\begin{array}{r}4,36 \\
17,09\end{array}$ \\
\hline $\begin{array}{l}\text { English et Bay- } \\
\text { ley, } 1978\end{array}$ & $\begin{array}{l}\text { Somerset En- } \\
\text { gland }\end{array}$ & $\left\{\begin{array}{l}\text { C. glareolus } \\
\text { A. sylvaticus }\end{array}\right.$ & $\begin{array}{r}77 \\
132\end{array}$ & $\begin{array}{r}42 \\
2\end{array}$ & $\begin{array}{r}9 \\
18\end{array}$ \\
\hline $\begin{array}{l}\text { Notre étude } \\
1986\end{array}$ & $\begin{array}{l}\text { Ouest-Bretagne } \\
\text { Massif cen- } \\
\text { tral }\end{array}$ & $\left\{\begin{array}{l}\text { C. glareolus } \\
\text { A. sylvaticus }\end{array}\right.$ & $\begin{array}{r}51 \\
146\end{array}$ & $\begin{array}{c}13,7 \\
2\end{array}$ & $\begin{array}{l}0 \\
2,7\end{array}$ \\
\hline
\end{tabular}


dont le mode de formation des aleuries, la mise en évidence parfois d'organes perforateurs, la pathogénicité de certaines espèces les rendent proches des dermatophytes. Si certaines souches ont été identifiées d'après leur forme conidienne (anamorphe) ou Chrysosporium d'autres ont développé en culture des gymnothèces (Arthroderma curreyi, Arthroderma cuniculi) ou des cleistothèces (Anixiopsis stercoraria).

\subsubsection{Chrysosporium keratinophilum (Frey), Carmichael, 1962.}

Ce kératinophile doué de pouvoir kératinolytique est isolé du sol du monde entier. Retrouvé aussi chez les petits mammifères sauvages (Gugnani et coll., 1975, Hubalek et coll., 1979) sa présence sur près d'un quart des lapins de garenne examinés dans notre étude est fait nouveau. 11 contamine aussi près de $12 \%$ des campagnols roussâtres. L'étude de Mariat et coll., qui fait référence en France (1976) ne le signalait pas. Ce champignon tellurique s'implante donc sur l'animal.

\subsubsection{Chrysosporium tropicum, Carmichael, 1962.}

Cette espèce proche de la précédente est un saprophyte du sol, sans pouvoir pathogène notable, isolé parfois du pelage des petits mammifères (Hubalek et coll., 1979) et du plumage des oiseaux (Chabasse et Guiguen, 1986 ; Hubalek, 1976 ; Sur et Ghosh, 1980).

2.2.3. Chrysosporium multifidum*, Dawson, 1963, anamorphe d'Arthroderma multifidum, Dawson, 1963.

Cette espèce, probablement cosmopolite, a été retrouvée dans le sol et les terriers de lapins; nous l'avons uniquement isolé chez Oryclolagus cuniculus.

2.2.4. Arthroderma curreyi*, Berkeley, 1860 et Arthroderma cuniculi, Dawson, 1963.

Ces espèces sont très proches. En l'absence de formes sexuées, les conidies de diamètre $\leqslant 4 \mu$ permettent de regrouper ces deux espèces avec Arthroderma ciferri dans le groupe Curreyi (Padhye et Carmichael, 1971).

Elles sont des saprophytes du sol et ont été aussi signalées sur le pelage des mammifères sauvages dans les terriers de lapins (Dawson, 1963) dans les nids et sur les plumes d'oiseaux (Pugh, 1964 ; Hubalek, 1976). Ces champignons kératinolytiques sont isolés avec une fréquence non négligeable dans notre étude en particulier chez le lapin de garenne pour $A$. curreryi. Nous avions retrouvé ces espèces sur le plumage des pigeons de ville à Bordeaux (Chabasse et Guiguen, 1986), elles sont rarement isolées du sol en France (Visset, 1975; Bourrat, 1986). Le pelage de ces mammifères représente donc un environnement tout à fait favorable.

2.2.5. Chrysosporium georgii* (Varsavsky et Ajello), Van Oorschot, 1980.

Ce champignon autrefois appelé Trichophyton georgiae, dont le stade sexué est Arthroderma ciferri (Varsavsky et Ajello, 1964) est classé maintenant parmi

\footnotetext{
* Espèce isolée pour la première fois sur le pelage des micromammifères et du lapin de garenne en France.
} 
les Chrysosporium en raison de l'absence de macroconidies véritables. Véritable kératinophile et kératinolytique, cette espèce est quelquefois citée dans la littérature comme un saprophyte du pelage de mammifères sauvages (Hubalek et coll., 1979) ou du plumage des oiseaux (Hubalek, 1970). Sa présence sur le poil du lapin de garenne dans près d'un tiers de nos prélèvements est un fait nouveau en France.

2.2.6. Chrysosporium pannorum* (Link), Hughes, 1958, Geomyces pannorum, Link; Sigler et Carmichael, 1976.

C'est une espèce cosmopolite largement répandue dans le sol. Si son pouvoir kératinolytique est limité, sa présence a une fréquence élevée aussi bien chez les micromammifères que chez le lapin dans des biotopes variés, témoigne de l'importance de son réservoir tellurique. Récemment, les auteurs japonais ont isolé de ce champignon un antibiotique : la chrysandine active sur les bactéries gram-négatives (Yamashita et coll., 1984).

\subsubsection{Chrysosporium merdarium* (Link), Carmichael, 1962.}

Ce kératinophile isolé du sol, du fumier et des excréments d'animaux a aussi été trouvé chez Apodemus sylvaticus par Hubalek et coll. (1979) en Yougoslavie. C'est un saprophyte non kératinolytique dénué, apparemment de tout pouvoir pathogène (Carmichael, 1962).

\subsubsection{Chrysosporium parvum*, Carmichael, 1962.}

Ce champignon qui n'attaque ni les cheveux, ni la cellulose sous sa forme saprophytique est pourtant l'agent de l'adiaspiromycose animale et humaine dans sa forme parasitaire (Boisseau-Lebreuil, 1972). Il est très rarement isolé sous sa forme mycélienne dans la nature (Ciferri et Montemartini, 1959). Sa découverte sur le pelage de quelques micromammifères du Cantal mérite d'être signalée. Le microclimat de cette région a été probablement favorable au développement de ces formes mycéliennes chez ces animaux fouisseurs.

\subsubsection{Anixiopsis stercoraria (Hansen), Hansen, 1897.}

L’identité de cette espèce est encore discutée, selon (Alpinis, 1968) le genre Anixiopsis, Hansen, 1897 est synonyme d'Aphanoascus fulvescens (Cooke), Alpinis, 1968. Sa forme conidienne serait un Chrysosporium proche de Chrysosporium keratinophilum. Isolé dans le monde entier, il se présente souvent comme un banal saprophyte (Gugnani et coll., 1975) sa pathogénicité est réelle, il est à l'origine d'infections cutanées animales et humaines (Rippon et coll., 1970 ; Vanbreuseghem, 1980 ; Albala et coll., 1982 ; Todaro et coll., 1984 ; Marin et Campos, 1984).

\section{Conclusion}

Si nos résultats confirment largement les travaux réalisés antérieurement, des faits nouveaux apparaissent. 


\section{LA MÉTHOdE UTILISÉE}

L'association des deux méthodes de prélèvement, sur un même animal permet d'isoler un nombre plus important et varié de champignons kératinophiles.

\section{LE NOMBRE LIMité DE DERMATOPHYTES POTENTIELlement ISOlÉS}

Trichophylon menlagrophyles est rarement retrouvé, uniquement chez Apodemus sylvalicus et Talpa europeae ; on peut aussi s'étonner de l'absence de Microsporum gypseum ; notons à ce sujet que Mariat et coll. (1976), n'avaient isolé cette espèce que dans $0,18 \%$ des 3782 animaux étudiés. Le rôle de ces mammifères dans la transmission de dermatophyties chez l'Homme est donc très limité.

Ia fréquence élevée de Trichophyton terrestre en particulier chez le lapin de garenne (dont la présence ne peut être expliquée par une simple contamination du sol) est peut-être, pour cette espèce dite géophile, une première étape vers le parasitisme chez l'animal (Chmel et Vollekova, 1981).

\section{L'ImPORTANCE DES CHAMPIGNONS VOISINS DES DERMATOPHYTES SOUVENT FOHTEMENT KÉHATINOPHILES}

En dehors de Scopulariopsis brevicaulis, ce genre Chrysosporium avec 10 espèces différentes est largement représenté.

Hormis Anixiopsis stercoraria incriminé de plus en plus dans des lésions cutanées chez l'Homme et Chrysosporium parvum, forme mycélienne saprophytique de l'agent de l'adiaspiromycose animale et humaine, ces champignons ont un pouvoir pathogène très limité. En effet, la plupart sont des saprophytes du milieu extérieur (sol, plantes, végétaux...). Certains ont déjà été décrits dans des biotopes assez spécifiques riches en kératine d'animaux, nids d'oiseaux, terriers de lapins, fumier d'origine animale. Leur présence sur le pelage de ces mammifères est-elle transitoire, liée à une simple contamination du sol ou au contraire assistet-on à un passage progressif du sol vers l'animal ? Ceci mérite d'être confirmé par un suivi régulier de ces espèces.

\section{BIBLIOGRAPHIE}

Albala I.., Moreda A. : Róle épidémiologique du lapin dans la transmission à l'Homme de Trichophyton mentagrophytes dans la région de Aragon (Espagne). Bull. Soc. Fr. Mycol. Med., 1981, $X, 21-23$.

Alteras 1., Nisterov Y., Ciolofax I. : The occurrence of dermatophytes in wild animals from Romania. Sabourandia, 1966, 4, 215-218.

Badillet G., Gillot B., Piétrini P., Espinosavillegas M. E. : Microsporum persicolor chez l'Homme et chez l'animal. Bull. Soc. Fr. Mycol. Med., 1972, I, 11-14.

Battell G., Biancheis W., Frigo P., Amorati A. I., Mantovani A., Pagliani A. : Survey of keratinophilic fungi in marmot (Marmola marmola) burrow soils and in adjoining soil. Sabouraudia, 1978, 16, 83-86.

Beaucounsu J. C., Launay H. : Le lapin de garenne el les zoonoses. Med. Mal In/ecl., 1977, $11,495-501$.

Boisseau-I.ebreyl M. T'. : Contribution à l'étude de l'adiaspiromycose cxpérimentale. Thèse Docloral 3e cycle, Université de Rennes, 1972, 117 p. 
Bourhat H. : I.es micromycètes kératinophiles telluriques dans la plaine du Forez. Thèse Doctorat en Pharmacie, Lyon, $1986,107 \mathrm{p}$.

Chabasse I)., De Bieve C., Covatahmanac'h A., Badillet G., Guiguen C. : Flore kératinophile isolée des micromammifères sauvages. Note préliminaire. Bult. Soc. Mycol. Med., 1984, $X I I I, 333-336$.

Chabasse D., Guiguex C. : Flore kératinophile isolée du plumage des pigeons de ville à Bordeaux. Bull. Soc. Fr, Mycol. Med., 1986, XV, 161-164.

Chabasse D., Launay H., ReEcht V. : Flore kératinophile isolée du lapin de garenne (Oryctolagus cuniculus Linne). Note préliminaire. Bull. Soc. Fr. Mycol. Med., 1986, XV, 165-168.

C.hmel I.., Buchyald J. : Small rodents as reservous of trichophytoses. Rec. Adv. Hum. Anim. Mycol. (ed. I. Chmel), 1967, 121-125. Publ. House Slovak. Acad. Sci., Brat islava.

Chmel L., Vol.tekova A. : Développement du parasitisme chez les champignons kératinophiles. Lyon Med., 1981, 245, 37-40.

Ciferri R., Montemartini A. : Taxonomy of Haplosporangium parbum. Mycopath. Mycol. Appl., 1959, 10, 303.

Dawson C. O.: Two new species of Arthroderma isolated from soil from rabbit burrow. Sabouraudia, 1963, 2, 185-191.

Dawson C. O. : Studies on certain keratinophilic fungi. Thesis Science, Iaculty, University of Glasgow Scotland, 1966.

1)URiE E. B., Frey O. : A new of Trichophylon from New South Wales. Mycologia, 1957, 49, 401-411.

ENGLISH M. P. : The ecology of some keratinophilic fungi associated with hedgehogs. $N$. $Z$. Med. $J ., 1964,63,586-591$.

Exglish M. P., Southers H. N. : Microsportum persicolor infection on a population of small wild mammals. Sabouraudia, 1967, 5, 302-309.

ENGLish M. P., BAYLEY J. A. : Dermatophytes in a population of bank voles and woodmice. Mycopathologia, 1978, 66, 67-71.

Evolceanu B., Alteras 1., Cojocanu I. : Considérations sur l'ubiquité du Trichophyton lerrestre. Durie et Frey, 1957. Saprophyte du sol. Son inoculabilité. Mycopathologia, 1962, 16, 35-46.

Garg A. P.. Sudha Gaviotra, MukerJi K. G., Pugh G. J. F. : Ecology of keratinophilic fungi. Proc. Indian Acad. Sci. (Plant Sci.), 1985, y4, 149-163.

Gextles .J. C., Dawsox C. O., Connole M. D. : Keratinophilic fungi on cats and dogs. Sabothraudia, $1965,4,171-175$.

Gugnani H. C., Wattal B. I.., Sanduu R. S. : Dermatophylis and other keralinophilic fungi recovered from small mammals in India. Mykosen, 1975, 12, 529-538.

Houin R., Rouget-Campasa Y., I.E Fichoux Y., Iancasthe F., Bazin J. C., Deniau M., Bozognisi J. : Isolement de Trichophyton mentagrophytes (Robin et Blanchard), 1896, Nannizia persicolor (Stokdale), 1967, et Trichophyton terrestre (Durie et Frey), 1957, du pelage des rongeurs. Essai d'interprétation écologique. Ann. Parasitol. Hum. Comp., 1972, 17, $421-429$.

Hubalek $Z$. : Occurrence of keratinolytic fungi in nests of tree sparrow ( $P$ asser montanus $\mathrm{L}$.) in relation to the substrate moisture. Ceska Mycol., 1976, 30, 106-109.

HubAlek Z. : Trichophyton georgiae Varsavsky and Ajello from birds in Czechoslovakia and Yugoslavia. Sabouraudia, 1970, 8, 1-3.

Hubalek Z., Rosicky B., Otcenasek M.: Fungi on the hair of small wild mammals in Czechoslovakia and Yugoslavia. Ceska Mykologi, 1979, 33, 81-93.

Kxudrson W. U., Ronenstad G. W. : The isolation of keratinophilic fungi from soil and wild animals in South Dakota. Mycopathologia, 1970, 40, 309-323.

Mantovani A. : The role of animals on the epidemiology of the mycoses. Mycopalhologia, 1978, $65,61-66$.

Mantovani A., Morganti I.., Battelli G., Mantovani A. L., Poglayen G., Tampieri M. P., Veccu G. : The role of wild animals in the ecology of dermatophytes and related fungi. Folia Parasilol. (Praha), 1982, 29, 279-284.

Mariat F., Chatelain J., Rouffaud M. A. : Étude sur la contamination par les champignons dermatophytes d'une population de petits mammifères sauvages en Alsace. Mycopathologia, $1976, ; 8,71-78$.

Marin G., Campos R.: Dermatofitosis por A phanoascus fulvescens. Sabouraudia, 1984, 22, 311-314.

MARPLES M. J. : Non domestic animals in New Zealand and in Parotonga as a reservoir of the agents of ringworm. N. Z. Med. J., 1967, 66, 299-302.

Mckeever S., Menges R. W., Kaplan W., AJello l.. : Ringworm fungi of feral rodents in South Western Georgia. Am. .J. Vet. Res., 1958, 19, 969-972.

Mexges R. W., Love G. I., Smith W. W., Geong I. K. : Ringworm in wild mammal in South Western Georgia. Am. J. Vet. Res., 1957, 18, 672-677.

OTCENASEK M., Dvorak J. : The isolation of Trichophyton lerrestre and other keratinophilic fungi from small mammals of South Eastern Moravia. Sabouraudia, 1962, 2, 111-113.

Otcenasek M., Hubalek Z., SixL M. : Survey of dermatophytes on hair of small mammals from Austria. Folia Parasitol., 1980, 1, 83-87.

PAdнye A. A., CarmichaEl J. W. : The genus Arthroderma Berkeley. Canadian J. Bot., 1971. 19, 1527-1540. 
PUGH G. J. F. : J)ispersal of A. curreyi by birds and its role in the soil. Sabouraudia, 1964, 3, $275-278$.

Rippox J. W., LEe I: C., McMillex S. : Dermatophyle infection caused by Aphanoascus fulvescens. Arch. Dermatol., 1970, 102, 552-555.

Scott I). W., Kirk R. W., Ventinck-Smith J. : Dermatophytosis due to Trichophyton terrestre infection in a dog and a cat. J. Am. Anim. Hosp. Ass., 1980, 16, 53-59.

Sur B., Grosh G. R. : Keratinophilic fungi from Orissa, India. II. Isolations from feathers of wild birds and domestic fowls. Sabouratdia, 1980, 18, 275-280.

Tonaro F., Criseo G., Unzi C. : A propos d'un cas de Tinea pedis du à Anixiopsis fulvescens (Cooke, de Vries). Bull. Soc. Fr. Mycol. Med., 1984, XIII, 239-242.

Vanbeuseghem R., De Vroey Ch. : Dermatophytic infection by Anixiopsis stercoraria in wild boar (Sus scrofa). Mykosen, 1980, 23, 183-187.

VArvasky E., AJello I.. : The perfect and impertect forms of nex keratinophilic fungus: Arthroderma ciferrii sp nov; Trichophyton georgii sp nov. Rev. Palh. Vegela., 1964, 4, 35 1-354.

Visset M. F. : Contribution à la recherche des champignons kératinophiles telluriques dans le massif armoricain et étude au microscope électronique à balayage. Thèse 3 e cycle Biol. Anim., Nantes, 1973, 130 p.

Yamashita M., Tsunumi Y., Hosoda J., Комohi T., Kohsada M., Imanaka H. : Chrysandin, a novel peptidyl nucleoside antibiotic I. Taxonomy, fermentation, isolation and characterisaion. J. Antibiot., 1984, 11, 1279-1283.

DFs Michoficies et des Michofilus de cette punlication peuvent hto obtenus aupres de

This publication is available in Wicroform from:

MASSON - SPPIF (réf. MIMC)

120, boulevard Saint-Germain, 75280 Paris Cedex 06

(C) Masson, Paris, 1987 .

Le Directeur de la Publication : J) J. Talamon

Tous droils de traduction, d'adaplation et de reproduction par tous procédés réservés pour tous pays.

La loi du 11 mars 1957 n'autorisant, aux termes des alinéas 2 et 3 de l'article 41, d'une part, que les a copıes ou reproductions strictement réservées à l'usage privé du copiste et non destinées à une utilisation collective v et, d'autre part, que les analyses et les courtes citations dans un but d'excmple et d'illustration, « toute représentation ou reproduction intégrale ou partielle, faite sans le consentement de l'auteur ou de ses ayants droit ou ayants cause, est illicite "(alinéa $1^{\text {er }}$ de l'article 40 ).

Cette représentation ou reproduction, par quelque procédé que ce soit, constituerait donc une contrefaçon sanctionnée par les articles 425 et suivants du Code pénal.

\begin{tabular}{|c|c|c|c|}
\hline Masson, éditeur, Paris. & Dépôt légal : 1987. & No d'ordre: 5861. & Seplembre 1987 \\
\hline
\end{tabular}

Printed in France. 